\title{
DISCUSSION
}

\section{Horizontal slice method of analysis}

\author{
M. SHAHGHOLI, A. FAKHER and C. J. F. P. JONES (2002). Géotechnique 51, No. 10,881-885
}

\section{J. Lam and K. S. Li, Victor Li \& Associates Ltd}

The authors have proposed the horizontal slice method for analysing the seismic stability of reinforced soil slopes. The idea of dividing the soil mass into horizontal slices appears to have been first proposed by Lo \& Xu (1992). The horizontal slice method developed by Lo \& Xu (1992) has the following advanced features:

(a) It incorporates the approach by Morgenstern \& Price (1965) in prescribing the interslice forces, and in achieving a rigorous solution that can satisfy force and moment equilibrium for all slices.

(b) It incorporates various techniques developed by $\mathrm{Li} \&$ White (1987) for slope stability calculations, including the unified solution scheme in simplifying the calculations for the method of slices, the compound iterative scheme in speeding up the convergence rate for calculation of factor of safety, and the rational polynomial technique for obtaining a rigorous solution and searching for the critical slip surface.

(c) It enables the magnitudes of individual reinforcing forces to be evaluated by considering strain compatibility between the soil and reinforcement along the rupture surface. The moment equilibrium of individual slices with reinforcement could thus be analysed.

(d) It can model the collapse condition controlled by the reinforcing elements reaching their limiting strain.

(e) The model enables the analysis to incorporate nonlinear load extension response with embedment and non-uniform distribution of reinforcement.

( $f$ ) The model enables the stress dilatancy characteristics of the soils to be incorporated in the analysis.

The method by Lo \& Xu (1992) can easily be extended for pseudo-static analysis of the seismic stability of reinforced soil slopes or walls. The horizontal slice method developed by Lo \& Xu (1992) is considered to be very versatile, and much more advanced than the simplified method proposed by the authors, whereby moment equilibrium for individual slices cannot be satisfied.

In Tables 1 and 3 of the paper, the authors attempt to summarise the number of unknowns in the vertical or horizontal slice method and the number of equations available for solving for those unknowns. Both tables appear to be incorrect. For $N$ slices there can be only $3 N$ independent equations based on force and moment equilibrium. The three equations for overall force and moment equilibrium mentioned in Table 1 of the authors' paper are not independent of the $3 N$ equations for individual slices, and will not lead to any new equations for solving for the unknowns. The number of unknowns for the horizontal slice method given in Table 3 of the paper has been underestimated.

Figure 1a shows the unknowns for both the vertical and horizontal slice methods. As indicated in Table 7, the numbers of equations and unknowns are the same for both methods. If the normal force at the base, $N_{i}$, is assumed to be at the mid-point of the slice base, which is the usual assumption justifiable for thin slices, the total number of unknowns will drop to $5 N-2$. The unknowns outnumber the available equations by $N-2$, as discussed in Li (1986, 1992). If, in addition, the $N-1$ vertical stresses are assumed to be equal to the overburden stress, as proposed by the authors, the total number of unknowns for the horizontal method of slices will drop further to $4 N-1$ and not $4 N$ as stated in Table 3 of the paper. The authors have made one assumption more than necessary in their simplified method. This will give rise to internal inconsistency among the basic equations available for solving for the unknowns. The end result is that the simplified method cannot completely satisfy moment equilibrium for individual slices.

The authors have summarised the limitations of available vertical slice methods. In the simplified Janbu's method and Bishop's method, the vertical interslice force $H_{i}$ is assumed to be zero at the interslice boundary. A total of $N-1$ assumptions have been made in these simplified methods because there are $N-1$ interslice boundaries. The simplified Janbu's method and Bishop's method therefore both prescribe one assumption more than necessary. Therefore, only the force or moment equilibrium for one slice cannot be satisfied, but not all slices as stated in Table 2 of the authors' paper.

The authors have compared the results of the horizontal slice method with the calculations by the program Reslope. It is not clear whether Reslope adopts a limit equilibrium method that satisfies both force and moment equilibrium. For the vertical slice method, published results by Fredlund \& Krahn (1977), Li (1986, 1992) and Li \& White (1987) indicate that methods based on force equilibrium are generally sensitive to the assumption about the interslice forces. Before jumping to the conclusion that the simplified horizontal slice method proposed by the authors is accurate, it is prudent to check whether the simplified method does give similar results to those of other rigorous horizontal slice methods, such as that of Lo \& Xu (1992).

\section{Authors' reply}

The authors would like to thank the discussers for their comments and contribution about the horizontal slice method of analysis proposed by the authors. The comments made can be grouped into two categories: general comments about previous works, and specific comments about the proposed method.

\section{GENERAL COMMENTS ABOUT PREVIOUS WORKS}

The concept of dividing a soil mass into non-vertical slices (Juran et al., 1990) or horizontal slices has been published before, notably by Gutierrez \& Tatsuoka (1988). However, the assumptions and the simplicity of the solutions are different. The strain-base design method suggested by Lo \& Xu (1992) provides a good general approach for design but requires a large computational effort. It could be extended for pseudo-static analysis but the computational effort would increase. In contrast, the horizontal slice method (HSM) proposed by the authors is simple to apply. Lo and $\mathrm{Xu}$ (1992) mention a number of advantages of their method, which can incorporate other concepts, such as 

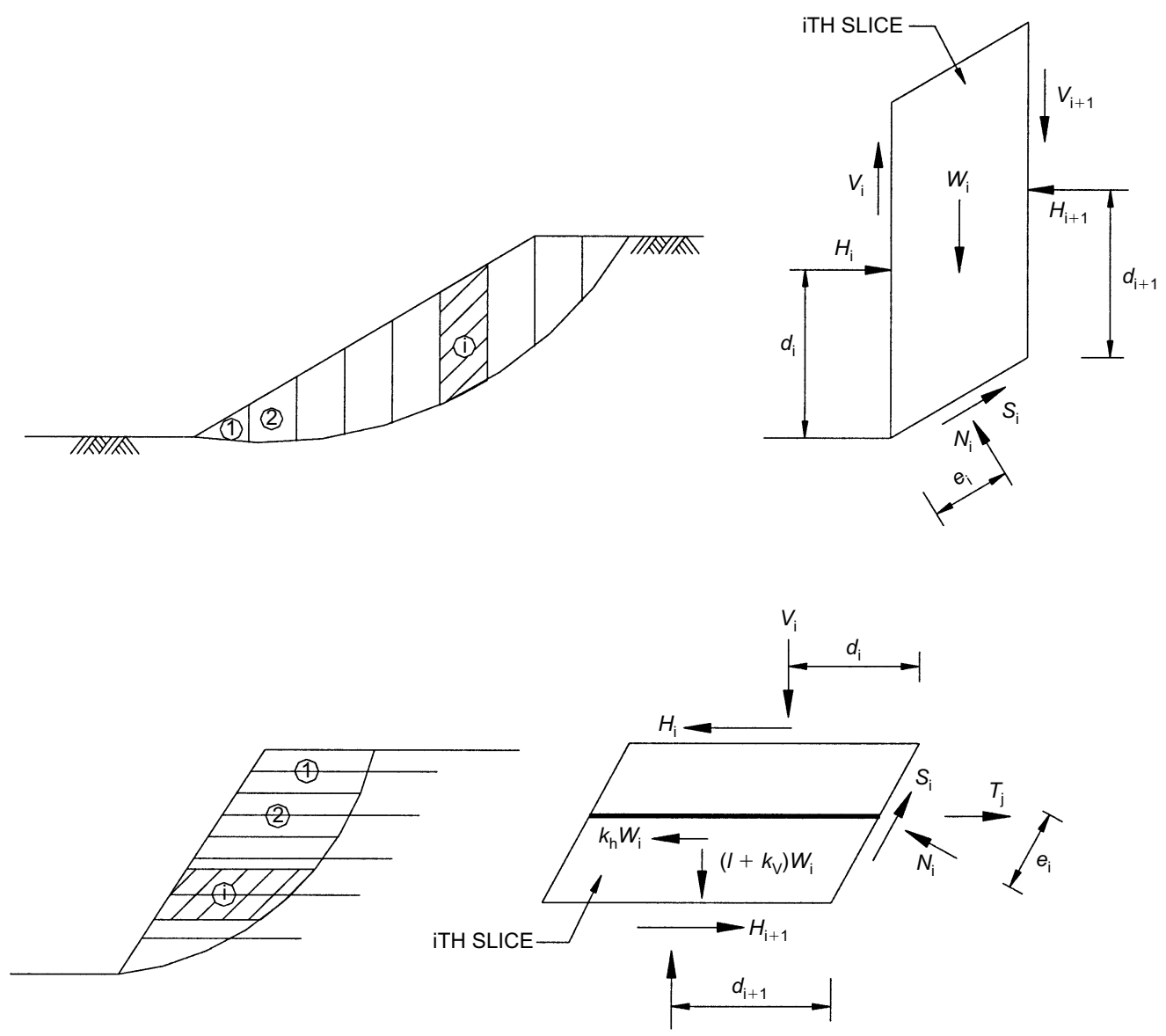

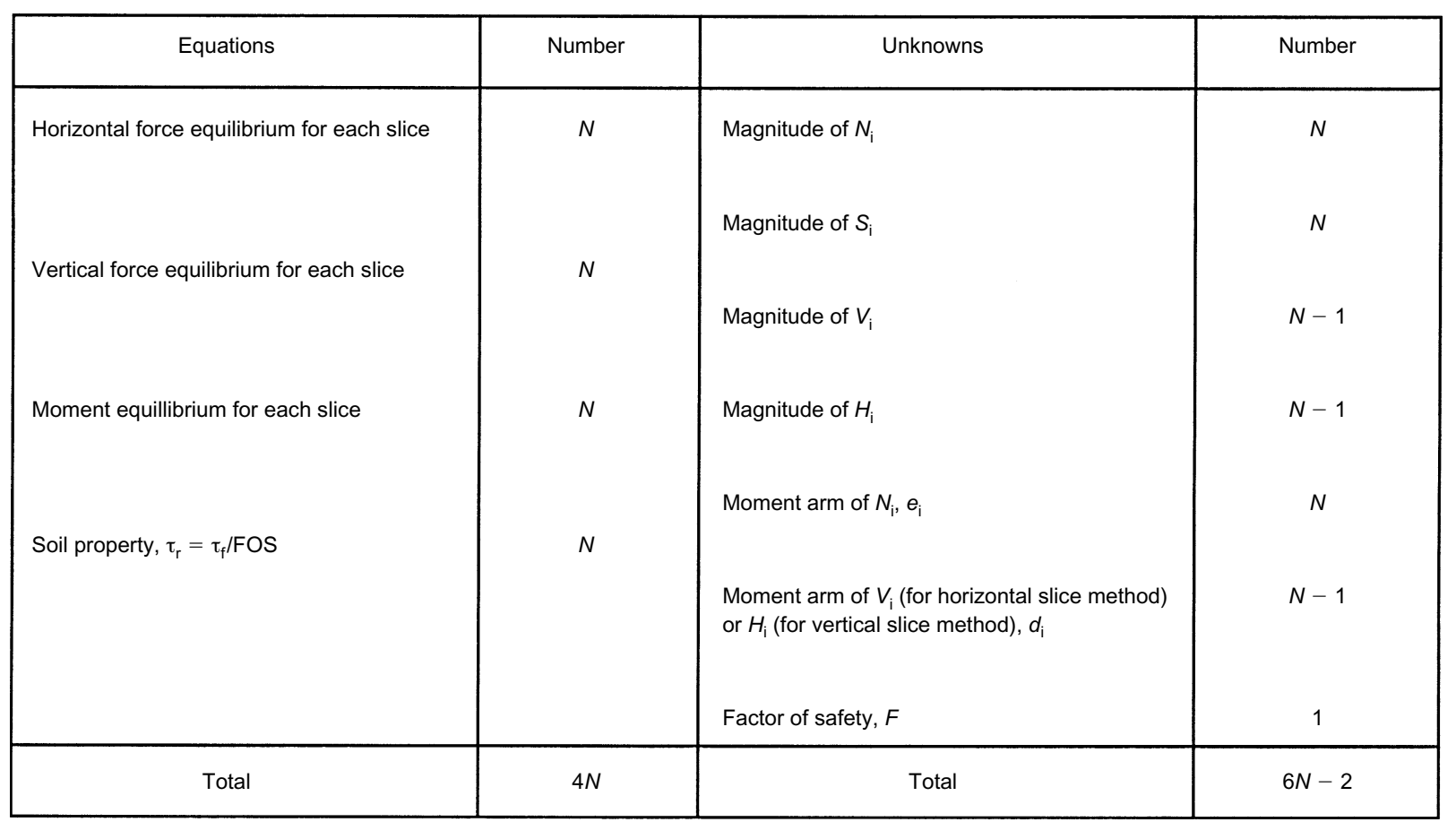

Fig. 1a. Forces acting on (a) a Vertical Slice and (b) a Horizontal Slice

Morgenstern \& Price's (1965) approach. These concepts can be incorporated into other methods, including HSM.

The number of governing equations for the vertical slice method, as stated in Table 1 of the paper, is correct. The three equations for overall force and moment equilibrium might not be independent for some specific assumed slip surfaces, but they are generally independent and can be used for analysis. The limitations of Janbu's and Bishop's methods, as detailed in Table 2 of the paper, have been identified by Sharma (1991). 


\section{SPECIFIC COMMENTS ABOUT THE AUTHORS' PROPOSED METHOD}

Figure 1a of the discussion presents the number of unknowns in the HSM. The discussers state that the total number of unknowns is equal to $6 N-2$. Then they assume that the normal forces at the base are at the mid-point of each slice so that the number of unknowns drops to $5 N-2$. In addition, the discussers state that the vertical stresses are equal to the overburden stresses, as proposed by the authors, so that the magnitude of vertical interslice forces, $V_{i}$, can be calculated and the number of unknowns is further reduced to $4 N-1$. The discussers therefore conclude that the authors have made one assumption more than necessary. This conclusion is a result of the mistreatment of the related assumptions. The authors assume only that the vertical stresses are equal to the overburden pressure. This assumption means that the magnitudes of $V_{i}$ and the moment arms of $V_{i}$ are known. Accordingly the number of unknowns drops to $4 N$. This can be illustrated using Table 7 provided by Lo \& Xu. According to Table 7:

(a) Total number of unknowns $=6 \mathrm{~N}-2$.

(b) Number of vertical interslice forces, $V_{i}=N-1$.

(c) Number of moment arms of $V_{i}=N-1$.

Therefore the number of unknown following the authors' assumptions will be equal to

$$
(6 N-2)-(N-1)-(N-1)=4 N
$$

The $4 N$ available equations provide the solution, and there are no unnecessary assumptions.

Lo \& Xu (1992) also comment on the validation of the HSM proposed by the authors. As stated in the technical note, the analysis of a typical reinforced soil wall using the method is presented and compared with the results produced by Reslope (Leschinsky, 1997). The comparison is not presented as a final conclusion about the validity of the method, but to illustrate that the HSM can provide a convenient and easily applied analysis of reinforced/unreinforced soil structures under seismic loads. In particular, different seismic accelerations at different heights of a soil structure can be modelled. The horizontal slice method is still being developed, and further findings will be published on completion of the research.

\section{REFERENCES}

Fredlund, D. G. \& Krahn, J. (1977). Comparison of slope stability methods of analysis. Can. Geotech. J. 14, April, 429-439.

Gutierrez, V. \& Tatsuoka, F. (1988). Role of facing in reinforcing cohesionless soil slopes by means of metal strips. Proceedings of the international geotechnical symposium on theory and practice of earth reinforcement (IS-Kyushu '88), Fukuoka, pp. 289-294.

Juran, I., Baudrand, G. \& Farrag, K. (1990). Kinematical limit analysis for design of soil-nailed structures. J. Geotech. Engng 116, No. 1, 54-72.

Li, K. S. (1986). A re-appraisal of Janbu's general procedure of slices. J. Hong Kong Instn Engrs, No. 7, July, 19-24.

Li, K. S. (1992). A unified solution scheme for slope stability analysis. Proc. 6th Int. Symp. Landslides, Christchurch 1, $481-486$.

Li, K. S. \& White, W. (1987). Rapid evaluation of the critical slip surface in slope stability problems. Int. J. Numer. Anal. Methods Geomech. 11, No. 5, 449-473.

Lo, S. C. \& Xu, D. W. (1992). A strain-based design method for the collapse limit state of reinforced soil walls or slopes. Can. Geotech. J. 29, October, 832-842.

Morgenstern, N. R. \& Price, V. R. (1965). The analysis of the stability of slip surfaces. Géotechnique 15, No. 1, 79-93. 